\title{
Avaliação das medidas de controle do HIV na atenção básica
}

\author{
Evaluation of HIV control measures in primary care
}

Evaluación de medidas de control del VIH em atención primaria

Clarissa Mourão Pinho ${ }^{1 *}$, Cynthia Angélica Ramos de Oliveira Dourado ${ }^{1}$, Morgana Cristina Leôncio de Lima' ${ }^{1}$, Thaís de Souza Maia², Juliany Fernanda Alves de Souza Silva², Ellen Lucena da Silva², Beatriz Raquel Lira da Fonsêca'2, Lays Miranda da Silva Cabral2 ${ }^{2}$, Ana Luísa Gulard Galvão Beserra da Silva ${ }^{2}$, Maria Sandra Andrade ${ }^{1}$.

\section{RESUMO}

Objetivo: Avaliar as medidas de controle do HIV na Atenção Básica. Métodos: Estudo descritivo, transversal, com abordagem quantitativa, realizado com 163 enfermeiros da rede de Atenção Básica à Saúde dos oito Distritos Sanitários do município de Recife-PE. Utilizou-se um questionário próprio para verificar o perfil sociodemográfico e profissional dos enfermeiros e um questionário validado, voltado para profissionais de saúde, visado a avaliação das atividades de controle do HIV/Aids na Atenção Básica. Resultados: Observase que $85,9 \%$ das unidades "sempre" realizam o pré-natal em momento oportuno e $95,1 \%$ "sempre" solicitam sorologias para HIV na primeira consulta. Capacitações específicas foram disponibilizadas, no último ano, para $47,2 \%$ dos profissionais. Destaca-se que $58,9 \%$ possuem "sempre" materiais para testagem rápida e apenas $35 \%$ possuem quantidade de preservativos suficientes para a demanda das unidades. Conclusão: As ações de controle do HIV mostram resultados satisfatórios no atendimento às gestantes. Fazem-se necessários maiores investimentos em capacitações e no estabelecimento do fluxo de fornecimento de preservativos e materiais de suporte para operacionalização das medidas de controle do HIV na Atenção Básica.

Palavras-chave: HIV, Síndrome da imunodeficiência adquirida, Atenção primária à saúde, Integralidade em saúde.

\begin{abstract}
Objective: Evaluate HIV control measures in Primary Care. Methods: This is a descriptive, cross-sectional study, with a quantitative approach, carried out with 163 nurses from the Primary Health Care network of the eight Sanitary Districts of the city of Recife-PE. A questionnaire was used to verify the sociodemographic and professional profile of nurses and a validated questionnaire for health professionals to assess the control activities of HIV/AIDS in Primary Care. Results: It is observed that $85.9 \%$ of the units "always" perform prenatal care at an opportune time and 95.1\% "always" request serology for HIV at the first consultation. Specific training was made available in the last year to $47.2 \%$ of professionals. It is noteworthy that $58.9 \%$ "always" have materials for rapid testing and only $35 \%$ have enough condoms to meet the demand of the units. Conclusion: HIV control actions show satisfactory results in assisting pregnant women. Greater investments are needed in training and in establishing the flow of supply of condoms and support materials for the operationalization of HIV control measures in Primary Care.
\end{abstract}

Keywords: HIV, Acquired immunodeficiency syndrome, Primary health care, Integrality in health.

\footnotetext{
1 Universidade de Pernambuco, Recife - PE. *E-mail: clarissa.mourao@hotmail.com

${ }^{2}$ Faculdade de Enfermagem Nossa Senhora das Graças (FENSG), Recife - PE.
} 
RESUMEN

Objetivo: Evaluar las medidas de control del VIH en atención primaria. Métodos: Estudio descriptivo, transversal, con enfoque cuantitativo, realizado con 163 enfermeras de la red de Atención Primaria de Salud de los ocho Distritos Sanitarios de la ciudad de Recife-PE. Se utilizó un cuestionario para verificar el perfil sociodemográfico y profesional de las enfermeras y un cuestionario validado para profesionales de la salud para evaluar las actividades de control del VIH/SIDA en atención primaria. Resultados: Se observa que el $85.9 \%$ de las unidades "siempre" realizan atención prenatal en el momento oportuno y el $95.1 \%$ "siempre" solicitan serología para VIH en la primera consulta. Se impartió formación específica en el último año al 47,2\% de los profesionales. Es de destacar que el $58.9 \%$ tiene materiales "siempre" para pruebas rápidas y solo el $35 \%$ tiene suficientes condones para satisfacer la demanda de las unidades. Conclusión: las acciones de control del VIH muestran resultados satisfactorios en la asistencia a mujeres embarazadas. Se necesitan mayores inversiones en capacitación y en establecer el flujo de suministro de condones y materiales de apoyo para la puesta en práctica de medidas de control del VIH en Atención Primaria.

Palabras clave: VIH, Síndrome de inmunodeficiencia adquirida, Atención primaria de salud, Integralidad en salud.

\section{INTRODUÇÃO}

No ano de 2014, o Ministério da Saúde (MS) propôs uma alteração no modelo de atendimento às Pessoas Vivendo com HIV (PVHIV), com a descentralização dos Serviços de Assistência Especializada (SAE), tornando a Atenção Primária à Saúde (APS) a porta de entrada e ordenadora do cuidado ao HIV/Aids. Entretanto, essa proposta não anula a atuação e importância do SAE, mas sugere que o cuidado seja compartilhado e integral entre esses dois serviços, sendo o SAE colocado como um serviço matricial para a APS (BRASIL, 2017a).

Essa proposta tem como objetivo garantir maior acessibilidade aos usuários do sistema de saúde e justifica-se pela mudança do perfil dessas pessoas e a disponibilização de esquemas antirretrovirais (ARV) mais simplificados. Para isso, deve ser assegurado a oferta de exames de HIV para todas as pessoas que busquem o serviço, confidencialidade dos resultados e atendimento humanizado. Com isso, haverá o aumento do número de diagnósticos precoces e início do tratamento em tempo oportuno (BRASIL, 2017a).

Para aqueles diagnosticados com HIV, recomenda-se a estratificação de risco em: assintomáticos e sintomáticos. Os assintomáticos são aqueles que não apresentam sinais e sintomas relacionados à doença. Já os sintomáticos serão aqueles que irão apresentar manifestações relacionadas à imunodeficiência decorrente da infecção pelo HIV.

Nesta perspectiva, é preconizado que os assintomáticos tenham seu atendimento na APS, sendo iniciado o tratamento com antirretrovirais de primeira linha imediatamente e os sintomáticos, co-infectados, gestantes, crianças, indivíduos com indicação de esquemas alternativos, sejam referenciados aos SAE. Além disso, deve ser disponibilizado exames de rotina, como contagem de linfócitos TCD4 e carga viral, qualificação profissional e suporte técnico através de serviço matricial (BRASIL, 2017a; BRASIL, 2017b).

Tais propostas justificam-se pela mudança do cenário da infecção pelo HIV, devido à disponibilização de esquemas ARV mais potentes e eficazes, com número de doses menores e efeitos colaterais mais leves. Estes aspectos contribuem para o aumento da adesão à TARV, proporcionando uma melhor adesão e qualidade de vida àqueles que convivem com o HIV. Sendo assim, a infecção pelo HIV deixa de ser considerada uma doença fatal e torna-se uma condição crônica, que ainda não possui cura, mas um tratamento eficiente (BOLLEN P, et al., 2015; SYED IA, et al., 2015).

Diante desse contexto, o processo de descentralização do atendimento ao HIV/Aids é ainda algo recente, havendo maior ênfase para os aspectos relacionados à testagem rápida. Contudo, a testagem rápida ainda se configura como um desafio dentro dos serviços de saúde. Algumas das dificuldades estão relacionadas ao 
déficit de funcionários, aumento da demanda de trabalho, falta de materiais e insumos, o que repercute negativamente na continuidade do serviço (BRASIL, 2015; ARAÚJO WE, et al., 2018).

Outros desafios estão relacionados à confiabilidade do teste rápido, sobrecarga de trabalho, equipes incompletas, falta de capacitações, falta de experiência profissional, expectativa de inexistência ou de elevada procura do teste, aspectos relacionados ao aconselhamento, comunicação no diagnóstico, oportunidades perdidas, falta de apoio pelos serviços matriciais (ZAMBENEDETTI G e SILVA RAN, 2016; ROCHA KB, et al., 2016).

Acredita-se que a atenção básica é fundamental para melhoria no atendimento das PVHIV, pois garante maior acesso e vínculo ao sistema de saúde, contribuindo para a melhoria na qualidade do atendimento a essas pessoas. Além disso, neste cenário é possível adotar estratégias de intervenções comportamentais, biomédicas e estruturais, também chamada de prevenção combinada, recomendadas pelo MS (BRASIL, 2017c).

Tal mudança talvez proporcione a continuidade da assistência, com a promoção do vínculo entre profissionais e pacientes, otimização das ações voltadas para educação em saúde, promoção, prevenção, diagnóstico precoce e início do tratamento do HIV/Aids em tempo oportuno, sendo possível um controle maior acerca da evolução da doença.

Dessa forma, torna-se relevante a condução de estudos que se aprofundem na avaliação das medidas de controle ao HIV adotadas na Atenção Básica. O estudo tem como objetivo avaliar as medidas de controle do HIV na Atenção Básica, sob a ótica dos enfermeiros.

\section{MÉTODOS}

Trata-se de um estudo descritivo, transversal, com abordagem quantitativa, realizado em 80 Unidades de Saúde da Família (USF) dos oito Distritos Sanitários (DS) do município de Recife-PE, no período de setembro de 2018 a abril de 2019.

A população foi composta por enfermeiros, de ambos os sexos, que compõem o quadro de funcionários das USF dos oito DS do município de Recife há pelo menos 1 ano. Foram excluídos aqueles que desempenhavam cargos de gestão e/ou coordenação da unidade.

Para o cálculo do tamanho da amostra foi levado em consideração a quantidade de USF no município de Recife-PE ( $n=276)$, uma estimativa de prevalência de $50 \%$ (desconhecida) e um intervalo de confiança (IC) de $95 \%$. Após o cálculo foi feita uma proporção amostral para cada estrato calculando o $\mathrm{n}$ proporcional e posteriormente foi feito à randomização simples de cada estrato, realizada através de sorteio simples.

O cálculo usado foi para estudos de população finita e variável dependente categórica, sendo expresso em, onde Za é o grau de confiança, geralmente expresso em 1,96 para 95\% de confiabilidade, pé a proporção de resultados favoráveis da variável na população, nesse estudo tomamos como base 0,4 , q é proporção de resultados desfavoráveis na população $(q=1-p), N$ é o tamanho da população (finita) e $E$ é o erro padrão, usualmente: $\pm 5 \%$ da proporção dos casos (precisão absoluta), ou $\pm 5 \%$ da média ( $1,05 \times$ média). Com esses dados chegou-se a um quantitativo amostral de 160 unidades, sendo distribuídas nos 8 Distritos Sanitários (DS) do município de Recife-PE da seguinte forma (Tabela 1). 
Tabela 1 - Distribuição do número de participantes de acordo com o número de Unidades de Saúde da Família e Equipes de Saúde da Família por Distrito Sanitário do município de Recife, Pernambuco, Brasil, 2019.

\begin{tabular}{cccccc}
\hline $\begin{array}{c}\text { Distritos } \\
\text { Sanitários } \\
\text { (DS) }\end{array}$ & No de USF & $\begin{array}{c}\text { No de equipes } \\
\text { por DS }\end{array}$ & $\begin{array}{c}\text { Frequência } \\
\text { proporcional }\end{array}$ & $\begin{array}{c}\text { Amostra } \\
\text { estratificada }\end{array}$ & $\begin{array}{c}\text { No de } \\
\text { enfermeiros } \\
\text { participantes }\end{array}$ \\
\hline DS I & 10 & 16 & 0,0057971 & 9,324837672 & 9 \\
DS II & 21 & 50 & 0,1811594 & 29,14011773 & 29 \\
DS III & 7 & 7 & 0,0253623 & 4,079616482 & 4 \\
DS IV & 20 & 40 & 0,1449275 & 23,31209418 & 23 \\
DS V & 17 & 33 & 0,1195652 & 19,2324777 & 19 \\
DS VI & 12 & 27 & 0,0978261 & 15,73566357 & 16 \\
DS VII & 21 & 48 & 0,173913 & 27,97451302 & 28 \\
DS VIII & 23 & 55 & 0,1992754 & 32,0541295 & 32 \\
\hline Total & 131 & 276 & 1,00 & 160,853 & 160 \\
\hline
\end{tabular}

Fonte: Pinho CM, et al., 2020.

As unidades foram ranqueadas em números crescentes de acordo com o quantitativo de USF por DS, sendo em seguida realizado um sorteio simples dessas. Posteriormente foi efetuado contato telefônico ou presencial com os enfermeiros de cada unidade para explicação do que se tratava a pesquisa e agendamento de dia e horário mais conveniente para a realização das entrevistas.

Para as unidades que os participantes se incluem nos critérios de exclusão, como, aqueles que desempenham cargo de gestão e/ou coordenação nas unidades estudadas ou que não aceitem participar do estudo, foi realizado novo sorteio de acordo com as unidades que não tenham sido contempladas ainda.

Antes do início de cada entrevista foi realizada a leitura o TCLE, onde constava os objetivos, riscos, benefícios do estudo, bem como os aspectos éticos relacionados e os direitos dos participantes. Logo após, foi solicitado a assinatura do TCLE em duas vias, ficando uma de posse do profissional e outra do pesquisador.

As entrevistas foram conduzidas em salas da própria unidade, de acordo com a disponibilidade, sendo aplicado um questionário sociodemográfico e profissional próprio e um instrumento validado para avaliação do controle do HIV na atenção básica.

O instrumento de avaliação do controle do HIV/Aids na atenção básica sob a ótica dos profissionais de saúde foi elaborado e validado (SILVA RAR, et al., 2017). O questionário é o único instrumento encontrado voltado para essa avaliação, sendo este considerado inédito. O mesmo possui 31 questões, voltadas a avaliação das medidas de controle ao HIV na Atenção Básica, sendo respondido através de uma escala tipo Likert (SILVA RAR, et al., 2017; CASTRO RR, 2015).

Os dados foram organizados em um banco de dados eletrônico por meio de digitação em planilha do Microsoft Excel (Office 2010), em seguida realizou-se correção e verificação dos erros de digitação do banco por meio da dupla validação. Após este processo, o banco foi exportado para a análise no programa StatisticalPackage for Social Science (SPSS) 20.0 e os resultados apresentados sob a forma de tabelas. A análise ocorreu por meio de inferência da estatística descritiva. 
Para avaliar a distribuição das variáveis quantitativas categóricas foram calculadas as frequências absolutas e relativas (percentuais) de cada variável e o teste de qui-quadrado $\left(X^{2}\right)$ foi utilizado para comparação das distribuições das proporções das categorias para cada variável pertinente para atender ao objetivo do estudo. Para as variáveis quantitativas, foram calculadas as medidas de tendência central e as medidas de dispersão, mínimo, máximo, média e desvio padrão.

Foram respeitados os preceitos éticos da Resolução 466/2012 do Conselho Nacional de Saúde e o estudo foi iniciado apenas após a aprovação do Comitê de Ética em Pesquisa (CEP) do Complexo Hospitalar HUOC/PROCAPE com o seguinte número de parecer: 2.224.605.

\section{RESULTADOS}

A amostra foi composta por 163 enfermeiros, que atuavam em 80 USF, distribuídos nos 8 DS do município de Recife, Pernambuco. A maioria do sexo feminino ( $90,2 \%)$, seguido do sexo masculino ( $9,8 \%)$. Com relação à idade, esta variou entre 25 a 69 anos, com média de $45,07 \pm 7,99$. A maior parte tinha entre 31 a 45 anos $(59,5 \%)$, seguido de 46 a 59 anos $(33,1 \%), \geq 60$ anos $(5,5 \%)$ e apenas $1,8 \%$ tinha menos de 30 anos de idade.

Quanto ao estado civil, a maioria se declarou casado (56,4\%), seguido dos solteiros (20,9\%), divorciados $(14,7 \%)$, em união estável $(4,3 \%)$ e viúvos $(3,7 \%)$. Houve a predominância daqueles que se declararam católicos $(60,1 \%)$, seguido dos evangélicos $(16,6 \%)$, espíritas $(13,5 \%)$, sem religião $(8,6 \%)$, umbandistas $(0,6 \%)$ e outras $(0,6 \%)$. Os testes de comparações de proporções foram significativos para todos esses fatores avaliados ( $p$-valor $<0,05)$.

Conforme à caracterização do perfil profissional, evidencia-se que o tempo de formação variou entre 4 a 42 anos, com média de 19,68 $\pm 7,25$ anos. Quanto aos anos de atuação na atenção básica, este variou entre 1 a 34 anos, com média de $15,77 \pm 6,29$ anos.

O que concerne ao quesito titulações acadêmicas, a maioria possuía pós-graduação em saúde da família $(63,2 \%)$, apenas $0,6 \%$ tinha pós-doutorado e $0,6 \%$ doutorado. Segundo à variável capacitação, a maioria declarou não ter recebido nenhuma capacitação relacionada à temática Infecções Sexualmente Transmissível, HIV/Aids (52,8\%), embora o teste de comparação de proporções não ter sido significativo para essa variável ( $p$-valor=0,481). Dos que responderam ter recebido algum tipo de capacitação no último ano, $33,7 \%$ fizeram sobre testagem rápida (Tabela 2). 


\section{Revista Eletrônica Acervo Saúde / Electronic Journal Collection Health | ISSN 2178-2091}

Tabela 2 - Caracterização profissional dos enfermeiros das Unidades de Saúde da Família do município de Recife, Pernambuco, Brasil, 2019.

\begin{tabular}{|c|c|c|c|}
\hline Fator avaliado & $\mathbf{N}$ & $\%$ & p-valor ${ }^{1}$ \\
\hline \multicolumn{4}{|l|}{ Anos de formação } \\
\hline até 15 anos & 56 & 34,4 & \multirow{3}{*}{0,001} \\
\hline 16 a 25 anos & 73 & 44,8 & \\
\hline$>25$ anos & 34 & 20,9 & \\
\hline \multicolumn{4}{|l|}{ Anos de atuação na atenção básica } \\
\hline Até 10 anos & 32 & 19,6 & \multirow{3}{*}{$<0,001$} \\
\hline De 11 a 20 anos & 103 & 63,2 & \\
\hline$>20$ anos & 28 & 17,2 & \\
\hline \multicolumn{4}{|l|}{ Titulações } \\
\hline Pós-Doutorado & 01 & 0,6 & \multirow{9}{*}{$<0,001$} \\
\hline Doutorado & 01 & 0,6 & \\
\hline Mestrado & 20 & 12,3 & \\
\hline Residência em outra área & 18 & 11,0 & \\
\hline Residência em saúde da família & 08 & 4,9 & \\
\hline Residência em saúde coletiva & 06 & 3,7 & \\
\hline Pós-graduação em saúde da família & 103 & 63,2 & \\
\hline Pós-graduação em saúde coletiva & 52 & 31,9 & \\
\hline Pós-graduação em outra área & 61 & 37,4 & \\
\hline \multicolumn{4}{|l|}{ Número de Pós-Graduação } \\
\hline Nenhuma & 01 & 0,6 & \multirow{4}{*}{$<0,001$} \\
\hline Uma & 73 & 44,8 & \\
\hline Duas & 63 & 38,7 & \\
\hline$>3$ & 26 & 16,0 & \\
\hline
\end{tabular}




\section{Revista Eletrônica Acervo Saúde / Electronic Journal Collection Health | ISSN 2178-2091}

\begin{tabular}{|c|c|c|c|}
\hline Possui outro vínculo & & & \\
\hline Sim & 101 & 62,0 & \multirow{2}{*}{0,002} \\
\hline Não & 62 & 38,0 & \\
\hline \multicolumn{4}{|l|}{ Onde? } \\
\hline Público & 89 & 54,6 & \multirow{4}{*}{$<0,001$} \\
\hline Particular & 9 & 5,5 & \\
\hline Docência & 3 & 1,8 & \\
\hline Não se aplica & 62 & 38,0 & \\
\hline \multicolumn{4}{|l|}{ Capacitações (último ano) } \\
\hline Sim & 77 & 47,2 & \multirow{2}{*}{0,481} \\
\hline Não & 86 & 52,8 & \\
\hline \multicolumn{4}{|l|}{ Qual? } \\
\hline Teste rápido (TR) & 55 & 33,7 & \multirow{4}{*}{$<0,001$} \\
\hline $\begin{array}{l}\text { Infecção Sexualmente Transmissível } \\
\text { (IST) }\end{array}$ & 19 & 11,7 & \\
\hline Outra & 3 & 1,8 & \\
\hline Nenhuma & 86 & 52,8 & \\
\hline
\end{tabular}

Legenda: ${ }^{1} p$-valor do teste Qui-quadrado $\left(X^{2}\right)$ para comparação de proporção.

Fonte: Pinho CM, et al., 2020. 
Acerca das ações de controle do HIV nas USF estudadas, os resultados a respeito das do uso correto do preservativo (Q21), demonstram que $30,7 \%, 27,6 \%$ e $26,4 \%$, relatam que as orientações ocorrem às vezes (2), frequentemente (3) e sempre (4), respectivamente.

Acerca da disponibilidade de materiais, espaço físico e conhecimento profissional, evidencia-se que em relação a disponibilidade de materiais (Q12) $18,4 \%$ dos enfermeiros relatam que as unidades não dispõem de materiais didáticos para a realização das atividades educativas e, 58,9\% afirmam que a unidade sempre tem disponível materiais para a realização dos TR (Q18); por sua vez apenas $35 \%$ dos profissionais declararam a disponibilidade de preservativos para atender a demanda da unidade (Q26).

Em relação à identificação da gestação (Q04), 85,9\% das USF realizam sempre (4) a consulta de pré-natal o mais rápido possível e 92,6\% afirmaram que os exames recomendados pelo Ministério da Saúde são solicitados sempre (4) na primeira consulta de pré-natal (Q11), nos quais a sorologia de HIV/Aids é solicitada sempre (4) por 95,1\% (Q28).

E na identificação do HIV/Aids pelas gestantes (Q15) 85,9\% sempre (4) são acompanhadas pela Unidade após serem referenciadas aos serviços especializados. Contudo, apenas $37,4 \%$ das gestantes recebem os resultados da $1^{\circ}$ e $2^{\circ}$ sorologia para HIV solicitadas durante o pré-natal (Q22).

Também, é importante destacar que $68,1 \%$ afirmam a oferta do teste rápido para os parceiros sexuais daqueles diagnosticados com HIV (Q06), contudo observa-se que 15,3\% responderam que essa testagem não é ofertada por estas unidades.

Outro aspecto, é acerca do acompanhamento das pessoas diagnosticadas com HIV, $40,5 \%$ e $28,8 \%$ (Q08), nessa ordem, relatam que esse acompanhamento ocorre sempre e frequentemente pelas unidades estudadas. Vale destacar que $71,2 \%$ dos profissionais sinalizam que realizam busca ativa dos parceiros sexuais (Q14) quando o diagnóstico do HIV/Aids for positivo.

Os testes rápidos são ofertados (Q30) sempre (4) em apenas 53,4\% das Unidades e a realização deles na própria Unidade básica acontece sempre (4) em 76,1\% dos casos. Na abordagem sindrômica as mulheres com sinais sugestivos e queixas ginecológicas (Q16), apenas em $46,6 \%$ das USF sempre (4) são ofertadas e realizadas a testagem rápida.

Quando questionado sobre a realização do aconselhamento pré-teste rápido para HIV/Aids (Q31) 20,2\% e 54\% dos partícipes responderam que o aconselhamento é realizado frequentemente (3) e sempre (4), respectivamente. A pesquisa aponta que $68,7 \%$ dos enfermeiros realizam o preenchimento da notificação. Assim, evidencia-se como entrave o não entendimento da obrigatoriedade do registro mediante a não totalidade das notificações (Quadro 1). 


\section{Revista Eletrônica Acervo Saúde / Electronic Journal Collection Health | ISSN 2178-2091}

Quadro 1 - Caracterização das ações de controle do HIV na Atenção Básica no município de Recife, Pernambuco, Brasil, 2019.

\begin{tabular}{|c|c|c|c|c|c|c|}
\hline Itens & $\begin{array}{c}0 \\
\text { Não }\end{array}$ & $\begin{array}{c}1 \\
\text { Raramente }\end{array}$ & $\begin{array}{c}2 \\
\text { Às vezes }\end{array}$ & $\begin{array}{c}3 \\
\text { Frequentemente }\end{array}$ & $\begin{array}{c}4 \\
\text { Sempre }\end{array}$ & p-valor ${ }^{1}$ \\
\hline $\begin{array}{l}\text { 1.Possui acesso a manuais e cadernos do Ministério da Saúde } \\
\text { referentes ao manejo de IST (Infecções Sexualmente Transmissíveis)? }\end{array}$ & $9(5,5 \%)$ & $3(1,8 \%)$ & $23(14,1 \%)$ & $36(22,1 \%)$ & $92(56,4 \%)$ & $<0,001$ \\
\hline $\begin{array}{l}\text { 2.Participou de treinamentos/capacitação sobre temas relacionados ao } \\
\text { controle do HIV/Aids nos últimos cinco anos? }\end{array}$ & $36(22,1 \%)$ & $26(16,0 \%)$ & $41(25,2 \%)$ & $31(19,0 \%)$ & $29(17,8 \%)$ & 0,363 \\
\hline $\begin{array}{l}\text { 3.São realizadas na área de abrangência da unidade de saúde, } \\
\text { campanhas informativas e de sensibilização acerca dos } \\
\text { comportamentos de risco para infecção por HIV? }\end{array}$ & $11(6,7 \%)$ & $14(8,6 \%)$ & $49(30,1 \%)$ & $50(30,7 \%)$ & $39(23,9 \%)$ & $<0,001$ \\
\hline $\begin{array}{l}\text { 4.Após a confirmação de gravidez por mulheres que procuram os } \\
\text { serviços de saúde é realizada a consulta pré-natal o mais rápido } \\
\text { possivel? }\end{array}$ & 0 & 0 & $1(0,6 \%)$ & $22(13,5 \%)$ & $140(85,9 \%)$ & $<0,001$ \\
\hline $\begin{array}{l}\text { 5.A unidade possui espaço físico disponível para a realização de } \\
\text { atividades educativas? }\end{array}$ & $61(37,4 \%)$ & $7(4,3 \%)$ & $19(11,7 \%)$ & $19(11,7 \%)$ & $57(35,0 \%)$ & $<0,001$ \\
\hline $\begin{array}{l}\text { 6.O teste rápido é ofertado aos parceiros de pessoas diagnosticadas } \\
\text { com HIV/Aids? }\end{array}$ & $25(15,3 \%)$ & $4(2,5 \%)$ & $3(1,8 \%)$ & $20(12,3 \%)$ & $111(68,1 \%)$ & $<0,001$ \\
\hline $\begin{array}{l}\text { 7.A entrega da camisinha (condon) acontece fora do espaço físico da } \\
\text { unidade de saúde? }\end{array}$ & $36(22,1 \%)$ & $18(11,0 \%)$ & $46(28,2 \%)$ & 24 (14,7\%) & 39 (23,9\%) & 0,003 \\
\hline $\begin{array}{l}\text { 8.As pessoas com diagnóstico positivo para HIV/Aids são } \\
\text { acompanhadas pela unidade? }\end{array}$ & $17(10,4 \%)$ & 7 (4,3\%) & $26(16,0 \%)$ & 47 (28,8\%) & $66(40,5 \%)$ & $<0,001$ \\
\hline $\begin{array}{l}\text { 9.Possui conhecimento do conteúdo dos manuais disponibilizados pelo } \\
\text { Ministério da Saúde a respeito das medidas utilizadas no controle do } \\
\text { HIV/Aids na atenção básica? }\end{array}$ & $9(5,5 \%)$ & $5(3,1 \%)$ & $31(19,0 \%)$ & $59(36,2 \%)$ & $59(36,2 \%)$ & $<0,001$ \\
\hline $\begin{array}{l}\text { 10.São realizadas campanhas informativas e de sensibilização para } \\
\text { prevenção por HIV/Aids nos equipamentos sociais sob a área de } \\
\text { abrangência da unidade de saúde? }\end{array}$ & $10(6,1 \%)$ & $16(9,8 \%)$ & $62(38,0 \%)$ & $42(25,8 \%)$ & $33(20,2 \%)$ & $<0,001$ \\
\hline
\end{tabular}




\section{Revista Eletrônica Acervo Saúde / Electronic Journal Collection Health | ISSN 2178-2091}

\begin{tabular}{|c|c|c|c|c|c|c|}
\hline $\begin{array}{l}\text { 11. Quando o resultado do teste de gravidez é positivo são solicitados } \\
\text { os exames recomendados pelo Ministério da Saúde na primeira } \\
\text { consulta? }\end{array}$ & $2(1,2 \%)$ & $1(0,6 \%)$ & $1(0,6 \%)$ & $8(4,9 \%)$ & $151(92,6 \%)$ & $<0,001$ \\
\hline $\begin{array}{l}\text { 12.A unidade disponibiliza de materiais didáticos para realização de } \\
\text { ações educativas? }\end{array}$ & 30 (18,4\%) & $21(12,9 \%)$ & $70(42,0)$ & $15(9,2 \%)$ & 27 (16,6\%) & $<0,001$ \\
\hline $\begin{array}{l}\text { 13.As pessoas com diagnóstico positivo para HIV/Aids são } \\
\text { referenciadas pela unidade? }\end{array}$ & $1(0,6 \%)$ & $1(0,6 \%)$ & 0 & $14(8,6 \%)$ & $147(90,2 \%)$ & $<0,001$ \\
\hline $\begin{array}{l}\text { 14.É realizada busca ativa aos parceiros (as) quando o diagnóstico do } \\
\text { HIV/Aids foi positivo? }\end{array}$ & $10(6,1 \%)$ & $3(1,8 \%)$ & $11(6,7 \%)$ & $23(14,1 \%)$ & $116(71,2 \%)$ & $<0,001$ \\
\hline $\begin{array}{l}\text { 15.As gestantes identificadas com HIV/Aids que foram referenciadas a } \\
\text { serviços de média e alta complexidade são acompanhadas pela } \\
\text { unidade de saúde? }\end{array}$ & $4(2,5 \%)$ & 0 & $1(0,6 \%)$ & $18(11,0 \%)$ & $140(85,9 \%)$ & $<0,001$ \\
\hline $\begin{array}{l}\text { 16.O teste rápido sorológico de HIV é solicitado a mulheres que } \\
\text { apresentam queixas sugestivas de infecção ginecológica? }\end{array}$ & $20(12,3 \%)$ & $3(1,8 \%)$ & $36(22,1 \%)$ & $28(17,2 \%)$ & 76 (46,6\%) & $<0,001$ \\
\hline $\begin{array}{l}\text { 17.Pessoas sugestivas de infecção por HIV que procuram a unidade } \\
\text { básica de saúde tem a oportunidade de realizar o teste de diagnóstico } \\
\text { na rede? }\end{array}$ & $2(1,2 \%)$ & 0 & $7(4,3 \%)$ & $30(18,4 \%)$ & $124(76,1 \%)$ & $<0,001$ \\
\hline $\begin{array}{l}\text { 18.A unidade de saúde disponibiliza de material para realização dos } \\
\text { testes rápidos para HIV/Aids? }\end{array}$ & $28(17,2 \%)$ & $2(1,2 \%)$ & $6(3,7 \%)$ & $31(19,0 \%)$ & $96(58,9 \%)$ & $<0,001$ \\
\hline $\begin{array}{l}\text { 19.Recebe/Recebeu capacitação para realizar os testes rápidos para } \\
\text { HIV/Aids? }\end{array}$ & $14(8,6 \%)$ & $9(5,5 \%)$ & $15(9,2 \%)$ & $24(14,7 \%)$ & $101(62,0)$ & $<0,001$ \\
\hline $\begin{array}{l}\text { 20.É realizada educação em saúde acerca de hábitos de vida saudáveis } \\
\text { nos equipamentos sociais sob área de abrangência da unidade? }\end{array}$ & $10(6,1 \%)$ & $10(6,1 \%)$ & $49(30,1 \%)$ & $53(32,5 \%)$ & $41(25,2 \%)$ & $<0,001$ \\
\hline $\begin{array}{l}\text { 21.Na entrega da camisinha (condon) é realizada orientação para seu } \\
\text { uso? }\end{array}$ & $14(8,6 \%)$ & $11(6,7 \%)$ & $45(27,6 \%)$ & $50(30,7 \%)$ & $43(26,4 \%)$ & $<0,001$ \\
\hline $\begin{array}{l}\text { 22. O resultado da } 1^{\circ} \text { e } 2^{\circ} \text { sorologia para HIV, solicitado no pré-natal é } \\
\text { entregue a gestante ainda durante a gravidez? }\end{array}$ & $5(3,1 \%)$ & $8(4,9 \%)$ & $40(24,5 \%)$ & $49(30,1 \%)$ & $61(37,4 \%)$ & $<0,001$ \\
\hline
\end{tabular}

REAS/EJCH | Vol.12(8) | e3462 | DOI: https://doi.org/10.25248/reas.e3462.2020 Página 10 de 14 


\section{Revista Eletrônica Acervo Saúde / Electronic Journal Collection Health | ISSN 2178-2091}

\begin{tabular}{|c|c|c|c|c|c|c|}
\hline $\begin{array}{l}\text { 23.São realizadas ações educativas coletivas voltadas para a } \\
\text { população relacionada à prevenção das IST (infecções sexualmente } \\
\text { transmissíveis)? }\end{array}$ & $1(0,6 \%)$ & $11(6,7 \%)$ & $66(40,5 \%)$ & $59(36,2 \%)$ & $26(16,0 \%)$ & $<0,001$ \\
\hline $\begin{array}{l}\text { 24.São realizadas ações educativas para informação e prevenção das } \\
\text { IST (infecções sexualmente transmissíveis) no espaço físico da unidade } \\
\text { de saúde? }\end{array}$ & $4(2,5 \%)$ & $12(7,4 \%)$ & $59(36,2 \%)$ & $60(36,8 \%)$ & $28(17,2 \%)$ & $<0,001$ \\
\hline $\begin{array}{l}\text { 25. É realizada a notificação de IST (infecções sexualmente } \\
\text { transmissíveis) e agravos no SINAN (sistema de informação de agravos } \\
\text { de notificação)? }\end{array}$ & 0 & $3(1,8 \%)$ & $4(2,5 \%)$ & $44(27,0 \%)$ & $112(68,7 \%)$ & $<0,001$ \\
\hline $\begin{array}{l}\text { 26. A quantidade de camisinha (condon) que a unidade recebe por mês } \\
\text { é suficiente para atender a demanda? }\end{array}$ & $18(11,0 \%)$ & $7(4,3 \%)$ & $29(17,8 \%)$ & $52(31,9 \%)$ & $57(35,0 \%)$ & $<0,001$ \\
\hline $\begin{array}{l}\text { 27. É realizada busca ativa às pessoas cujo diagnóstico do HIV foi } \\
\text { positivo e não retornaram para receber o resultado? }\end{array}$ & $7(4,3 \%)$ & $2(1,2 \%)$ & $1(0,6 \%)$ & $27(16,6 \%)$ & $126(77,3 \%)$ & $<0,001$ \\
\hline $\begin{array}{l}\text { 28. Na primeira consulta de pré-natal é solicitada a sorologia para } \\
\text { HIV/Aids? }\end{array}$ & $3(1,8 \%)$ & $1(0,6 \%)$ & 0 & $4(2,5 \%)$ & $155(95,1 \%)$ & $<0,001$ \\
\hline $\begin{array}{l}\text { 29.As ações educativas acerca do HIV/Aids são desenvolvidas sem } \\
\text { dificuldades/entraves? }\end{array}$ & $12(8,0 \%)$ & $12(8,0 \%)$ & $58(35,6 \%)$ & $42(25,8 \%)$ & 37 (22,7\%) & $<0,001$ \\
\hline $\begin{array}{l}30.0 \text { teste rápido ou sorológico para HIV é ofertado aos usuários da } \\
\text { unidade? }\end{array}$ & $18(11,0 \%)$ & $4(2,5 \%)$ & $16(9,8 \%)$ & $38(23,3 \%)$ & $87(53,4 \%)$ & $<0,001$ \\
\hline 31.Realiza aconselhamento pré-teste rápido para HIV/Aids? & $29(17,8 \%)$ & $6(3,7 \%)$ & $7(4,3 \%)$ & $33(20,2 \%)$ & $88(54,0)$ & $<0,001$ \\
\hline
\end{tabular}

Legenda: ${ }^{1} p$-valor do teste Qui-quadrado $\left(X^{2}\right)$ para comparação de proporção.

Fonte: Pinho CM, et al., 2020. 


\section{DISCUSSÃO}

Os dados coletados ao longo deste estudo evidenciam um perfil de profissionais de enfermagem em sua maioria do sexo feminino, na faixa etária entre 31 e 45 anos, casados e católicos. Mais de $60 \%$ dos participantes possuem mais de 16 anos de formação e pouco mais de $80 \%$ têm 11 anos de atuação na atenção básica.

Evidencia-se a busca dos enfermeiros pela saúde pública, incluindo a atenção básica. Os motivos estão relacionados a resolutividade do trabalho do enfermeiro, relacionamento interpessoal com a equipe e com a comunidade, autonomia, remuneração financeira e reconhecimento, o que proporcionam a este profissional sentimentos de prazer, satisfação, alegria e bem-estar no ambiente de trabalho (ROCHA GSA, et al., 2019).

Acerca das titulações observa-se a predominância daqueles com pós-graduação em saúde da família, seguido de saúde coletiva. Sobre a modalidade stricto sensu, apenas 20 possuíam mestrado, 1 doutorado e 1 pós-doutorado. Ainda, a maioria dos entrevistados possuíam outro vínculo, sendo estes com vínculo público. A necessidade de duplo vínculo está relacionada aos baixos salários pagos àclasse, contudo a necessidade de ter outro emprego colabora com o desgaste do profissional, sofrimento psíquico, adoecimento e até mesmo a morte entre os enfermeiros (DIAS MO, et al., 2019).

Sobre o atendimento ao HIV na atenção básica, estudos demonstram que a atuação do profissional é influenciada por diversos fatores, a saber: moral, ética, estigma, sigilo relacionado ao diagnóstico dos usuários, falta de habilidade técnica e manejo clínico, déficit na educação permanente dos profissionais, processo de trabalho e organizacional interno e externo, estrutura física adequadas, garantia de insumos e fortalecimentos de políticas públicas.

A partir do enfrentamento dos desafios frente a assistência prestadaàs PVHIV, possibilita aumento na qualidade do cuidado desempenhados pelas equipes que compõem a APS (MELO EA, et al., 2018; ZAMBENEDETTI G e SILVA RAN, 2016). As dificuldades relatadas dos profissionais na presente pesquisa é percebida pela oscilante variação de enfermeiros no início da vida profissional e da necessidade de educação permanente em saúde que possibilita a manutenção do conhecimento para o manejo clínico, possibilitando uma melhor qualidade na assistência com iniciativas qualificadas (BRASIL, 2018).

$O$ estudo verificou um déficit na promoção de momentos de capacitação e aprofundamento dos profissionais acerca de ISTs, HIV/Aids, limitando-se principalmente ao treinamento para a realização de TR, o que na prática evidencia a necessidade de intensificar o apoio e a promoção de programas de educação voltados ao tema, com o intuito de ampliar o acesso ao diagnóstico precoce, possibilitando o início do tratamento em tempo oportuno (BONES AANS, et al., 2018).

Outro ponto evidenciado foi a falta de recursos disponíveis nas unidades para a realização de ações de educação em saúde, aspecto muito importante para que o indivíduo tenha autonomia e opte pela forma de prevenção que melhor se adeque à sua realidade. Outro momento oportuno para orientação sobre o uso de métodos de prevenção são os de aconselhamento, que através da abordagem profissional permite que o indivíduo lide com bloqueios, e avalie suas reais possibilidades de risco de infecção, além de refletir e decidir por medidas preventivas viáveis em busca de uma melhor qualidade de vida, independente da sua condição sorológica.

O grande desafio é desenvolver e realizar abordagens individuais e em grupos, considerando as individualidades apresentadas, de forma a contemplar expectativas, necessidades do indivíduo ou grupo. (Secretaria de estado da saúde de São Paulo, 2017)

Nas unidades contempladas pelo estudo, a situação com relação à oferta de preservativos em quantidade, tamanho e tipo adequados, demonstrou-se por vezes insuficiente para atender as demandas dos territórios. No Brasil, o Departamento Nacional de DST, AIDS e Hepatites Virais (DDAHV), em conjunto com programas do governo local, adota-se como estratégia o reforço nesse aprovisionamento para ampliar o acesso universal e gratuito aos preservativos, visando a redução a ocorrência de infecções sexualmente transmissíveis (PINTO VM, et al., 2018; BRASIL, 2019). 
No contexto da APS a atenção àsaúde da mulher está diretamente ligada às consultas de prevenção ginecológica e aos serviços de pré-natal. O Ministério da Saúde recomenda o início do pré-natal no primeiro trimestre, seis consultas para uma gestação a termo e a realização de exames laboratoriais para ISTs, dentre eles a sorologia para HIV, visando diminuir a morbidade e a mortalidade materno-infantil e a transmissão vertical, uma vez que a identificação do risco gestacional permite a orientação e os encaminhamentos adequados. (NUNES JT, et al., 2016).

Ao longo do pré-natal a gestante deve ser orientada sobre a importância da testagem e os benefícios do diagnóstico precoce para a prevenção da transmissão vertical. A testagem para HIV deve ser realizada no primeiro trimestre, e solicitada preferencialmente na primeira consulta do pré-natal, e novamente no início do terceiro trimestre de gestação, devendo ser refeita em qualquer outro momento em que haja exposição de risco. O que foi observado ao longo do desenvolvimento desse estudo é que, apesar de haver a solicitação e a realização do exame, o laboratório municipal não suporta a demanda e os exames não ficam prontos em tempo hábil, dificultando as medidas de prevenção à transmissão vertical (BRASIL, 2019).

Nas USF incluídas no estudo foi possível observar um dado alarmante: em relação à abordagem sindrômica, os testes rápidos foram realizados em mulheres que apresentam sinais sugestivos e queixas ginecológicas em menos da metade das unidades, o que representa um obstáculo para o diagnóstico precoce.Adotar como prática no serviço a testagem de todas as mulheres que apresentem sinais ou queixas sugestivas de ISTs pode representar um grande avanço para o diagnóstico antecipado, aconselhamento adequado, pré e pós teste, início do tratamento, busca ativa pelos parceiros sexuais e, portanto, interrupção da cadeia de transmissibilidade. Além disso, é importante destacar que a subnotificação e a notificação tardia dificultam a identificação de áreas endêmicas e prejudicam as ações de vigilância em saúde (BRASIL, 2015; MELO MAS, et al., 2018).

Salienta-se como estratégia para melhora na assistência à saúde ao paciente com HIV no contexto da APS o enfrentamento e a readequação, que tem como objetivo superar os déficits e obstáculo já relatados no presente estudo através da readequação estrutural e prática. Destaca-se a importância da escuta ativa na investigação das práticas sexuais dos pacientes e aconselhamento pré-teste, bem como, promover a educação em saúde, provendo apoio emocional, educativo, avaliação de risco e auto reflexão, com a conscientização dos métodos de prevenção e uso consistente do preservativo (TAQUETTE SR, et al., 2017).

\section{CONCLUSÃO}

Com desenvolvimento do estudo, evidenciou-se aspectos positivos acerca do diagnóstico em gestantes, contudo, observa-se a necessidade de medidas de controle mais efetivas quanto a realização da TR a demanda espontânea, em parceiros sexuais, mulheres com sinais sugestivos e notificação de todos os casos diagnosticados, além da oferta de atividades educativas voltadas a prevenção das IST/HIV e disponibilização de preservativos em número adequado. Faz-se necessário maiores investimentos a respeito da disponibilização de recursos materiais, estruturais e humanos, com capacitações contínuas quanto a TR. Tais medidas têm como objetivo o êxito do processo de descentralização e uma assistência de qualidade no que tange a prevenção, diagnóstico e tratamento do HIV e demais IST.

\section{REFERÊNCIAS}

1. ARAÚJO WJ, et al. Percepção de enfermeiros executores de teste rápido em Unidades Básicas de Saúde. Rev. Bras. Enferm., 2018; 71(1): 631-636.

2. BRASIL. Ministério da Saúde. Secretaria de Vigilância em Saúde. Departamento de Vigilância, Prevenção e Controle das Infecções Sexualmente Transmissíveis, do HIV/Aids e das Hepatites Virais. Boletim Epidemiológico HIV/Aids; 2017.

3. BRASIL. Ministério da Saúde. Secretaria de Vigilância em Saúde. Departamento de Vigilância, prevenção e controle das Infecções Sexualmente Transmissíveis, do HIV/Aids e das Hepatites Virais. Cinco passos para a prevenção combinada ao HIV na Atenção Básica. Brasília: Ministério da Saúde; 2017.

4. BRASIL. Ministério da Saúde. Secretaria de Vigilância em Saúde. Departamento de Vigilância, Prevenção e Controle das Infecções Sexualmente Transmissíveis, do HIV/Aids e Hepatites Virais. Cuidado Integral às pessoas vivendo com HIV pela atenção básica. Manual para equipe multiprofissional; 2015. 
5. BRASIL. Ministério da Saúde. Secretaria de Vigilância em Saúde. Departamento de Doenças de Condições Crônicas e Infecções Sexualmente Transmissíveis. Protocolo Clínico e Diretrizes Terapêuticas para Prevenção da Transmissão Vertical do HIV, Sífilis e Hepatites Virais / Ministério da Saúde, Secretaria de Vigilância em Saúde, Departamento de Doenças de Condições Crônicas e Infecções Sexualmente Transmissíveis. - Brasília: Ministério da Saúde, 2019. 248.

6. BRASIL. Ministério da Saúde. Perfil da enfermagem no Brasil: relatório final: Brasil. Rio de Janeiro: NERHUS - DAPS - ENSP/Fiocruz, 2017; 721.

7. BRASIL. Ministério da Saúde. Secretaria de Gestão do Trabalho e da Educação na Saúde. Departamento de Gestão da Educação na Saúde. Política Nacional de Educação Permanente em Saúde: o que se tem produzido para o seu fortalecimento? / Ministério da Saúde, Secretaria de Gestão do Trabalho e da Educação na Saúde, Departamento de Gestão da Educação na Saúde - 1. ed. rev. - Brasília: Ministério da Saúde, 2018; 73.

8. BOLLEN $P$, et al. Clinical pharmacokinetics and pharmacodynamics of dolutegravir used as a single tablet regimen for the treatment of HIV-1 infection. Expert OpinDrugSaf., 2015; 14(9): 1457-72.

9. BONES AANS, et al. A educação para o enfrentamento da epidemia do HIV. Interface - Comunicação, Saúde, Educação, FapUNIFESP (SciELO), 2018; 22(1): 1457-1469.

10. CASTRO RR. Elaboração e validação de questionário para avaliação do controle do HIV/Aids na atenção básica. Dissertação (Mestrado em saúde e sociedade). Universidade do Estado do Rio Grande do Norte, Rio Grande do Norte, 2015.

11. DIAS MO, et al. Percepção das lideranças de enfermagem sobre a luta contra a precarização das condições de trabalho.Rev. esc. enferm. USP, 2019; 53: e03492.

12. MELO EA, et al. Cuidado, HIV/Aids e atenção primária no Brasil: desafio para a atenção no Sistema Único de Saúde? Rev Panam Salud Publica, 2018; 42:e151.

13. MELO MAS, et al. Percepção dos profissionais de saúde sobre os fatores associados à subnotificação no Sistema Nacional de Agravos de Notificação (Sinan). Revista de Administração em Saúde, 2018; 18(71): 1-17.

14. NUNES JT, et al. Qualidade da assistência pré-natal no Brasil: revisão de artigos publicados de 2005 a 2015. Cadernos Saúde Coletiva, FapUNIFESP, 2016; 24(2): 252-261.

15. PINTO VM, et al. Fatores associados às infecções sexualmente transmissíveis: inquérito populacional no município de São Paulo, Brasil. Ciência \& Saúde Coletiva, 2018; 23(7): 2423-2432.

16. ROCHA GSA, et al. Sentimentos de prazer no trabalho das enfermeiras na atenção básica.Rev. Bras. Enferm., 2019; 72(4): 1036-1043.

17. ROCHA KB, et al. Transversalidade a rede: o matriciamento na descentralização do aconselhamento e teste rápido para HIV, sífilis e hepatites. Saúde em debate, 2016; 40(109): 22-33.

18. SECRETARIA DE ESTADO DA SAÚDE DE SÃO PAULO. Diretrizes para implementação da rede de cuidados em IST/HIV/AIDS - Manual de Prevenção CRT - DST/AIDS. CCD., Secretaria de Estado da Saúde de São Paulo, 2017.

19. SILVA RAR, et al. Questionário para avaliação do controle do HIV/Aids na atenção básica. Acta Paulista Enferm., 2017; 30(3): 271-9.

20. SYED IA, et al. Adverse drug reactions and quality of life in HIV/Aids patients: Advocacy on valuation and role of pharmacovigilance in developing countries. HIV \&AIDS Review, 2015; 14(1): 28-30.

21. TAQUETTE SR, et al. Percepção de pacientes com AIDS diagnosticada na adolescência sobre o aconselhamento pré e pós-teste HIV realizado. Ciência \& Saúde Coletiva, 2017; 22(1): 23-30.

22. ZAMBENEDETTI G, SILVA RAN. Descentralização da atenção em HIV-AIDS para atenção básica: tensão e potencialidades. Physis [online], 2016; 26(3): 785-806. 